\title{
Entry requirements
}

Kaposi's sarcoma-associated herpesvirus (KSHV) causes Kaposi's sarcoma, which originates in endothelial cells. Hahn et al. identified ephrin type-A receptor 2 (EPHA2) as a putative KSHV receptor through co-immunoprecipitation experiments with the KSHV envelope glycoproteins $\mathrm{H}$ and $\mathrm{L}(\mathrm{gH}-\mathrm{gL}$ ), which are required for cell entry of the virus, and KSHV virions. EPHA2 was found to be crucial for KSHV infection of endothelial cells, as overexpression of EPHA2 increased KSHV entry into cells, and KSHV infection was inhibited by various strategies that blocked access to or reduced expression of EPHA2. Furthermore, $\mathrm{gH}-\mathrm{gL}$ binding to EPHA2 resulted in phosphorylation of EPHA2 and endocytosis, which is known to be involved in KSHV entry. In human Kaposi's sarcoma samples, EPHA2 expression correlated with KSHV infection.

These data corroborate the recent finding that EPHA2 is required for entry and trafficking of KSHV in human microvascular dermal endothelial cells. Chakraborty et al. found that EPHA2 co-immunoprecipitated with integrins (which have been implicated in viral entry) in KSHV-infected cells. KSHV was observed to activate EPHA2 and amplify cellular signalling and viral endocytosis. Furthermore, KSHV entry was reduced by EPHA2 knockdown or treatments that blocked binding to EPHA2. They also found that the tyrosine kinase inhibitor dasatinib inhibited KSHV entry.

As ongoing lytic viral infection occurs in Kaposi's sarcoma lesions, these papers indicate that EPHA2 is a potential therapeutic target in this disease. 